\title{
Colon capsule endoscopy in colorectal cancer screening: Interim analyses of randomized controlled trial CareForColon2015
}

\section{(ㄷ)(1)}

Authors

Ulrik Deding ${ }^{1,2}$, Thomas Bjørsum-Meyer ${ }^{1,2}$, Lasse Kaalby ${ }^{1,2}$, Morten Kobaek-Larsen ${ }^{1,2}$, Marianne Kirstine Thygesen ${ }^{1,2}$, Jeppe Buur Madsen ${ }^{3}$, Rasmus Kroijer ${ }^{4}$, Gunnar Baatrup ${ }^{1,2}$

Institutions

1 Department of Surgery, Odense University Hospital, Svendborg, Denmark

2 Department of Clinical Research, University of Southern Denmark, Odense, Denmark

3 Department of Biochemistry and Immunology, University Hospital Lillebaelt, Vejle, Denmark

4 Department of Surgery, Hospital of Southwest Jutland, Esbjerg, Denmark

submitted 30.3.2021

accepted after revision 22.6.2021

Bibliography

Endosc Int Open 2021; 09: E1712-E1719

DOI 10.1055/a-1546-8727

ISSN 2364-3722

(c) 2021. The Author(s).

This is an open access article published by Thieme under the terms of the Creative Commons Attribution-NonDerivative-NonCommercial License, permitting copying and reproduction so long as the original work is given appropriate credit. Contents may not be used for commercial purposes, or adapted, remixed, transformed or built upon. (https://creativecommons.org/licenses/by-nc-nd/4.0/)

Georg Thieme Verlag KG, Rüdigerstraße 14,

70469 Stuttgart, Germany

Corresponding author

Ulrik Deding, University of Southern Denmark, Department of Clinical Research, Winsløwparken 19, Odense 5230, Denmark ulrik.deding@rsyd.dk

\section{ABSTRACT}

Background and study aims The Danish CareForColon2015 trial, launched in 2020 as part of the Danish Colorectal Cancer Screening program, is the largest randomized controlled trial to date on colon capsule endoscopy (CCE). This paper presents the interim analysis with the objective of ensuring the safety of patients in the intervention group and evaluating the clinical performance of the trial's predefined clinical parameters.

Patients and methods We evaluated the initial 234 CCEs according to quality, safety, and completion. The participation rates and preference distribution of all individuals invited were analyzed and sample size calculations were adjusted.

Results Fecal immunochemical test and diagnostic participation rates were $62.1 \%$ and $91.1 \%$, respectively. The completion rate for CCEs was $67.9 \%$ and the rate of conclusive investigations was $80.3 \%$. The polyp detection rate (PDR) was high $(73.5 \%)$, only two $(0.85 \%)$ technical failures in 234 videos were observed, and six suspected cancers were identified (2.6\%). No major adverse events were recorded. The required number of invitations had been underestimated due to inaccurate assumptions in sample size calculations.

Conclusions The trial was efficient and safe in terms of CCE quality and time to diagnostic investigation. Participation rates and PDRs were high. The proportion of suspected cancers was lower than expected and will be followed. The completion rate for CCEs was acceptable but lower than expected and the CCE procedure was reviewed for potential improvements and Resolor was added to the regime. The number of invitations for the intervention group of the trial has been adjusted from 62,107 to 185,153 .

\section{Introduction}

In August 2020, the CareForColon2015 trial [1] (CFC2015) was launched in Southern Denmark. It is a randomized controlled trial (RCT) testing colon capsule endoscopy (CCE) as a filter test for colorectal cancer (CRC) screening. The trial will include 2015 patients undergoing CCE investigations. The primary aim of the trial is to compare the number of detected cancers and intermediate- and high-risk adenomas between the intervention and control groups. In addition, the CFC2015 trial has a number of secondary aims, including patient acceptability, complication rate, completion rate, interval CRC rate, patientreported outcomes (PRO), long-term cancer incidence rate, social inequality, development of algorithms aiming to improve 
CCE applicability, and cost-effectiveness of the trial structure of the active group. The study protocol is described in detail elsewhere [1]. Leading up to the initiation and development of the trial, a number of studies were published highlighting the potential of CCE. The observed benefits ranged from increased screening participation [2], reduced patient discomfort [3], to increased detection of high-risk polyps [4].

The interim analysis was included in the study protocol to be conducted after inclusion of 200 CCE investigations [1]. It was carried out to ensure efficient and safe procedures in the intervention group of the study, as compared to the usual practice. The process also allows for modifications to the study.

The CFC2015 protocol defines a number of focus areas for interim analysis. These include quality of CCE investigations, participation rate, preference distribution, completion rate, and sample size considerations. Parameters were defined in the study protocol, dictating potential consequences and/or necessary actions from the results of the interim analysis. These include a revision of the information material and invitation procedure, if the intervention group participation rate is below $60 \%$, and a revision of capsule delivery and bowel preparation procedures, if the CCE completion rate was between $65 \%$ and $80 \%$. Further, the trial will be terminated if the participation rate proves to be lower than $55 \%$ or if the CCE completion rate is below $65 \%$. Moreover, the number of invitations will be recalculated from the interim results to ensure that the final inclusion is accurate and realistic.

The aim of this study was to conduct the interim analysis of the CFC2015 trial, based on the predefined conditions, thereby investigating participation rate, preferred investigation type, CCE completion rate, and the quality of the CCE investigations. In addition, this study aimed to adjust the sample size calculations.

The RCT protocol was approved by the Regional Health Research Ethics Committee (Ref. S-20190100). The trial was further approved by the Danish data protection agency (Ref. 19/ 29858). The trial was registered with clinicaltrials.gov (Ref. NCT04049357).

\section{Patients and methods}

CRC screening in Denmark consists of a fecal immunochemical test (FIT) followed by colonoscopy if FIT hemoglobin exceeds $100 \mathrm{ng} / \mathrm{mL}$ [5]. CFC2015 randomizes individuals for regular CRC screening (control group) or screening with the free choice between colonoscopy and CCE following positive FIT (intervention group). Individuals in the intervention group are invited to indicate whether they prefer CCE or colonoscopy if FIT positive by adding a green or red sticker, respectively, to the sample tube. Individuals are referred for colonoscopy following CCE if a polyp larger than $9 \mathrm{~mm}$ or more than two polyps are found by the CCE. In cases in which no polyps are detected, the individual is subject to an 8-year quarantine from the CRC screening program. In the case of one to two polyps $\leq 9 \mathrm{~mm}$, the patient is invited to re-enter biennial screening and has the opportunity to submit a new FIT after 2 years. All others, including patients with incomplete CCE or at least one colonic segment with poor bowel preparation, are invited for colonoscopy. The study will be concluded when 2,015 individuals have undergone CCE. Exclusion criteria included history of abdominal surgery (excl. appendectomy), symptomatic Crohn's disease, cardiac pacemaker, known renal insufficiency, pregnancy/breast feeding, allergy toward bowel preparation or booster medication, known severe constipation or inability to comply either cognitively or physically. For full design and methods of CFC2015, see the protocol [1]. These interim analyses were conducted 4 weeks after the first 200 CCE evaluations were performed. Data for analysis were extracted from research databases, from PRO questionnaires, and from internal participant lists. Information regarding each individual was extracted if his or her invitation was dated the same day or before the FIT invitation dates of the first 200 CCE participants. Data management and descriptive statistics, including proportions and rates, were conducted using SAS 9.4 (SAS, Gary, North Carolina, United States).

\section{Participation rates}

Participation rates were defined for two separate stages; FIT participation and diagnostic participation after a positive FIT.

FIT participation refers to the proportion of individuals invited for CRC screening who submit a FIT sample and will be investigated for the intervention group and the control group.

Diagnostic participation refers to the proportion of individuals with a positive FIT who participate in a following CCE or colonoscopy. Diagnostic participation will be estimated for the intervention group and for the subgroups of indicated preferred diagnostic procedure.

\section{Completion rates of colon capsule endoscopies}

Completion rates were defined in four ways; acceptable bowel preparation, complete transit, complete investigation, and conclusive investigation.

Acceptable bowel preparation refers to the proportion of individuals for whom the bowel preparation was rated fair or better on the Leighton-Rex scale [6] for each of the colonic segments reached by the capsule endoscope. Bowel preparation was evaluated for each of the colonic segments (cecum, right colon, transverse colon, left colon, and rectum) and all segments needed to be fair or better. Complete transit refers to the proportion of CCEs in which the output images visualize the hemorrhoidal plexus. Complete investigation rate refers to the proportion of CCEs in which the output images visualize the hemorrhoidal plexus and bowel preparation was rated fair or better on the Leighton-Rex scale for all colonic segments. Conclusive investigation refers to the proportion of CCEs in which either I) the output images visualize the hemorrhoidal plexus and bowel preparation was rated fair or better on the Leighton-Rex scale or II) the output images enabled conclusive identification of more than two polyps, at least one polyp $>9 \mathrm{~mm}$, or suspected cancer requiring either a diagnostic or therapeutic follow-up. 


\section{Quality of colon capsule endoscopies}

The quality of CCEs was to be evaluated according to four different characteristics; polyp findings, adverse events (AEs), time span, and technical issues.

Polyp findings refer to polyps and suspected cancers. Polyp findings were estimated as average number of polyps per individual, as the proportion of individuals with at least one finding (polyp detection rate [PDR]), and as the proportion of individuals with suspected cancer. Finally, polyp findings were estimated as the proportion of individuals with more than two polyps, at least one polyp $>9 \mathrm{~mm}$, or a suspected cancer lesion.

In addition to complications, AEs also refer to both major and minor events. They were divided into the categories: capsule retention, rectal bleeding, abdominal pain, and other. The "other" category gave participants the option to describe any experienced $\mathrm{AE}$ not covered by the categories. Participants were invited to complete an online questionnaire on their CCE experience 3 days after investigation.

Time span was defined in two ways: time to investigation and time to diagnostics. Time to investigation was calculated as days from the date of positive FIT until the date of conducted CCE. Time to diagnostics was calculated as days from the CCE investigation until the date on which the CCE video had been analyzed and the results registered in the patient file.

Technical failures can occur during CCE. The capsule transmits images in real time to a receiver worn on a belt by the participant. In case of connection timeout or other recorder dysfunctions, this was noted in the CCE report. The proportion of CCEs with technical failures leading to repeat CCE or colonoscopy was estimated.

\section{Sample size adjustments}

The assumptions used when calculating the required sample size (124,214 individuals) in order to recruit 2,015 individuals for CCE investigation were:

1. FIT-positive rate of $6.9 \%$

2. FIT participation rate of $66 \%$

3. Of individuals offered the choice between CCE and colonoscopy, $75 \%$ would choose CCE (preference proportion)

4. Dropout rate for CCE of $5 \%$

If any of these assumptions deviates significantly from reality, it will affect the number of invitations needed. The FIT-positive rate was calculated as the proportion of positive FITs among the samples that were submitted. FIT participation rate was estimated as described above and with at least 4 weeks of followup from distribution of the invitation. Preference proportion for CCE was calculated as the proportion of individuals in the intervention group who had indicated CCE as their preferred investigation by adding a green sticker to the sample tube. Preference proportion for colonoscopy and no preference indicated were also calculated. The dropout rate for CCE was defined as the proportion of individuals who dropped out from the study or for whom it became evident that they met exclusion criteria after they indicated their preference for CCE.

\section{Results}

Distribution of CRC screening invitations for the study began on August 3, 2020. On November 19, 2020, the 200th to $211^{\text {th }}$ CCE investigations were conducted. For the first 211 CCE participants, the latest CRC screening invitation was distributed on October 19, 2020. From August 3 to October 19, 46,242 screening invitations were distributed evenly between the intervention group and the control group. During follow-up, an additional 23 CCE investigations were concluded for individuals invited at the same date or before October 19; therefore, these analyses reflect 234 CCE investigations. The mean age was 62.4 years (range 50.3 to 74.9 ) and $56.2 \%$ of participants were male.

\section{Participation rate}

The FIT participation rates were $62.2 \%$ in the control group and $62.1 \%$ in the intervention group, respectively. In the intervention group, 14,461 individuals submitted a FIT sample, of whom, 1,432 (9.9\%) had marked the test tube with a red sticker, while 7,235 (50.0\%) had marked it with a green sticker. The remaining 5,794 (40.1\%) sample tubes were not marked. Eighty-six FIT-positive individuals who had marked with a green sticker dropped out ( $\triangleright$ Fig. 1 ). Mean age and gender distribution did not differ significantly between dropouts and participants. Overall, the diagnostic participation in the intervention group was $91.1 \%$. In subgroups of red, green, and no sticker, the diagnostic participation rates were $80.8 \%, 96.8 \%$, and $87.5 \%$, respectively ( $\triangleright$ Table 1 ).

\section{Completion rate of colon capsule endoscopies}

In total, 234 CCE investigations were conducted. In 184 (78.6\%), the bowel preparation was acceptable for segments reached by the capsule. In 199 investigations (85.0\%), the hemorrhoidal plexus was visualized within recording time, and in 159 $(67.9 \%)$, the hemorrhoidal plexus was visualized with fair or better bowel preparation. The CCE investigations were conclusive in 188 instances (80.3\%) (> Fig. 2).

\section{Quality of colon capsule endoscopies}

The quality of CCEs was measured in four areas: polyp findings, AEs, time span, and technical issues.

\section{Polyp findings}

The average number of polyps per individual was 2.4 in the 234 investigations (564 polyps in total). The average number of polyps in the 188 conclusive CCE investigations was 2.9 per individual (544 polyps in total). In 159 complete investigations with acceptable bowel preparation, the average was 2.7 (424 polyps in total). The PDR was $73.5 \%$ as at least one polyp of any size was found in 172 individuals. At least one polyp $>5 \mathrm{~mm}$ in largest diameter was found in 152 (65.0\%) CCE investigations and at least one polyp >9 mm in 97 (41.5\%) CCE investigations. More than two polyps, at least one $>9 \mathrm{~mm}$, or 


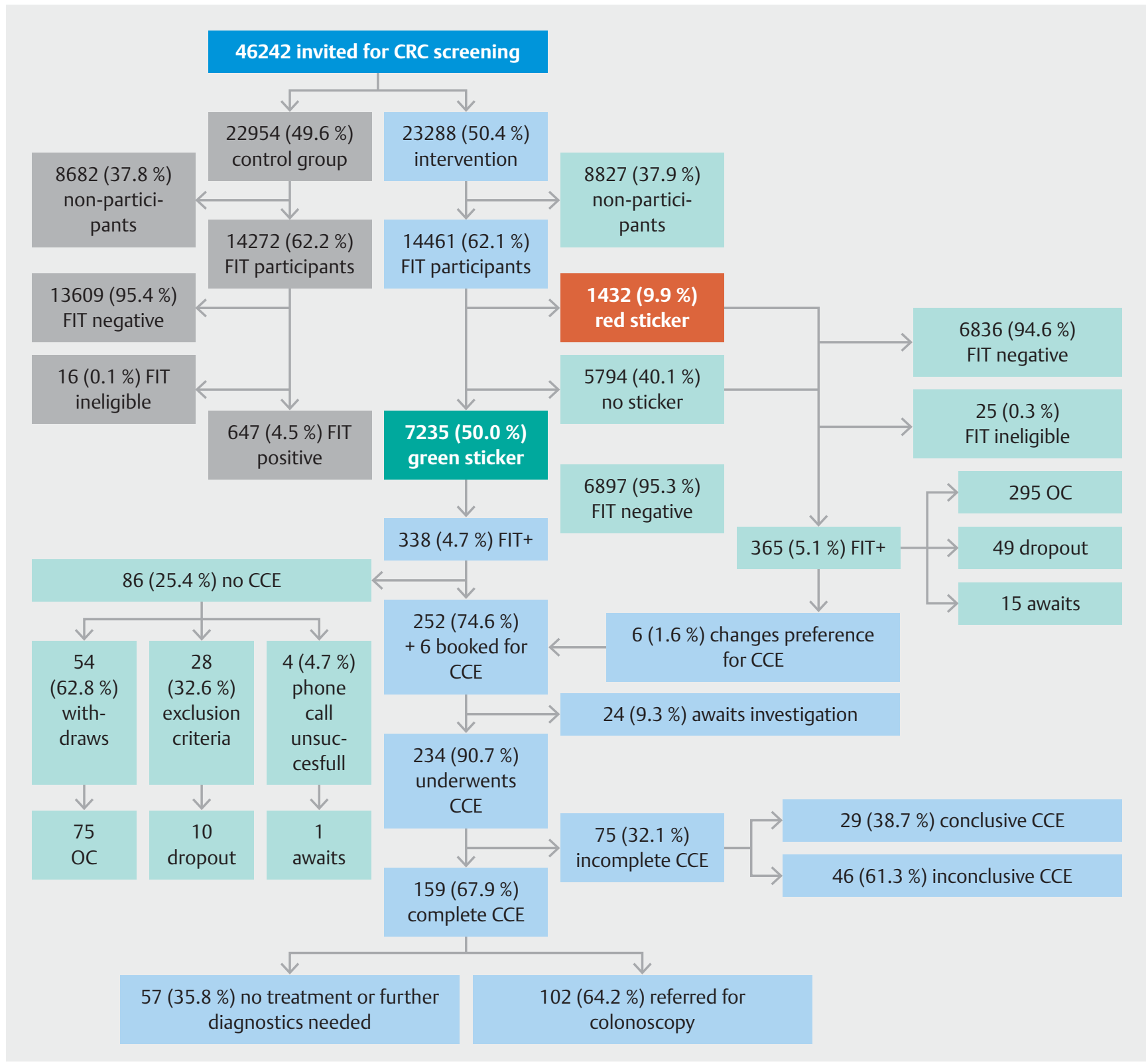

- Fig. 1 Flowchart of first 46,242 colorectal cancer screening invitations in CareForColon2015.

- Table 1 Diagnostic participation in the intervention group.

\begin{tabular}{|l|c|c|c|c|}
\hline & Red sticker & No sticker & Green sticker & Overall \\
\hline Participants & $63(80.8 \%)$ & $238(87.5 \%)$ & $303(96.8 \%)$ & $604(91.1 \%)$ \\
\hline Non-participants & $15(19.2 \%)$ & $34(12.5 \%)$ & $10(3.2 \%)$ & $59(8.9 \%)$ \\
\hline Awaiting investigation & 3 & 12 & 25 & 40 \\
\hline
\end{tabular}

a suspected cancer were identified in 126 of investigations $(53.8 \%)$. A total of six suspected cancers were identified between six individuals.

\section{Adverse events}

The questionnaire was successfully distributed to all 234 individuals and the response rate was $96 \%$ (225 participants). No capsule retentions or other major AEs were recorded. Minor AEs or discomfort were reported by 73 participants (31.2\%). When asked specifically, 27 (12\%) reported abdominal pain 


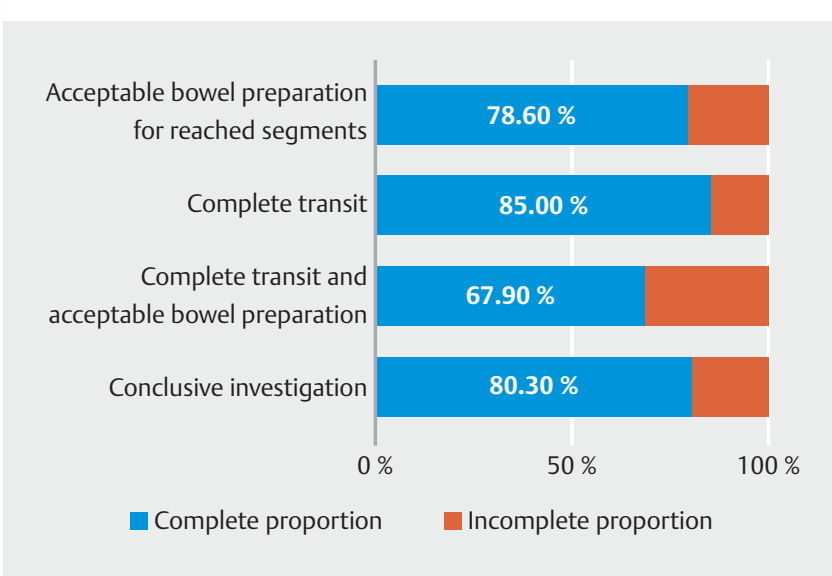

Fig. 2 Completion rates for colon capsule endoscopies.

and $19(8.2 \%)$ reported rectal bleeding. When asked whether they had experienced any other discomfort, 44 individuals $(19.6 \%)$ replied "yes" and added comments ( $>$ Table 2 ).

Time span and technical issues

The average time to CCE from positive FIT was 11.25 days. The average time to CCE diagnostics from day of capsule ingestion was 3.5 days.

A technical score was reported as a part of the CCE video evaluation. One investigation was deemed insufficient, four had short interruptions, and one had video transmission failure. In six instances, the technical score was not reported. The participant with video transmission failure from CCE was offered the choice of repeat CCE or colonoscopy, but chose not to undergo further diagnostics. Three of those with no reported technical video score were due to vomiting resulting in oral capsule excretion.

\section{Sample size adjustments}

For the first 14,461 individuals submitting a FIT sample in the intervention group, the FIT-positive rate was $4.9 \%$. The FIT-positive rate differed between preference groups in the intervention group. The green sticker group had a FIT-positive rate of $4.7 \%$, whereas in the red/no sticker groups, it was $5.1 \%$. This affects the sample size calculations, as it is needed to adjust the FIT-positive rate to $4.7 \%$ and to alter the order of calculations compared to the study protocol. The protocol includes a backwards calculation from 2,015 needed individuals multiplied by assumed CCE preference proportion and then FIT positivity rate. In the adjustment, we switched the two to achieve a precise estimate. The FIT participation rate was $62.1 \%$ in the intervention group (62.2\% in the control group). The preference proportion for CCE was $50.0 \%$, while $9.9 \%$ preferred colonoscopy, and $40.1 \%$ did not mark the tube. Eighty-six individuals (25.4\%) dropped out or met exclusion criteria after the first 338 FIT-positive individuals with an applied green sticker ( Fig.1). A summary of assumptions compared to observed proportions is shown in $>$ Table 3.

The power and sample size calculations conducted before the study launch had estimated a need for 124,214 individuals
- Table 2 Self-reported adverse events from colon capsule endoscopy.

\begin{tabular}{|l|c|}
\hline & Events (\%) \\
\hline Rectal bleeding & $19(8.2)$ \\
\hline Abdominal pain & $27(12.0)$ \\
\hline Other & $44(19.6)$ \\
\hline Physical pain from other parts of the body & $22(9.8)$ \\
\hline $\begin{array}{l}\text { Gastrointestinal discomfort (other than abdominal } \\
\text { pain) }\end{array}$ & $21(9.3)$ \\
\hline Affected energy levels or appetite & $5(2.2)$ \\
\hline Affected body temperature & $3(1.3)$ \\
\hline Affected balance, heart rhythm or psyche & $4(1.8)$ \\
\hline
\end{tabular}

- Table 3 Assumed and observed proportions for sample size calculations.

\begin{tabular}{|l|c|c|}
\hline & Assumption & $\begin{array}{c}\text { Observed } \\
\text { in interim }\end{array}$ \\
\hline FIT-positive rate & $6.9 \%$ & $4.7 \%$ \\
\hline FIT participation & $66.0 \%$ & $62.1 \%$ \\
\hline CCE preference proportion & $75.0 \%$ & $50.0 \%$ \\
\hline Dropout rate & $5.0 \%$ & $25.4 \%$ \\
\hline FIT, fecal immunochemical test; CCE, colon capsule endoscopy. \\
\hline
\end{tabular}

randomized for screening in the two groups, i.e. half in the intervention group. Applying these observed proportions to the calculations increased this number to 370,306 (185,153 for the intervention group) ( Fig.3), of which approximately 5.749 positive FIT are expected in the intervention arm.

\section{Discussion}

FIT participation was $62.1 \%$ and diagnostic participation was $91.1 \%$ in the intervention group of the study, which is similar to rates for the national CRC screening program at $60 \%$ and $90 \%$, respectively [7-9]. High participation rates are essential for the efficacy of population-based screening programs. In 21 European countries using the FIT-test in CRC screening settings, an overall participation rate of $49.5 \%$ was reported, which is somewhat below the European guideline reference cut-off of $65 \%$ [10].

The CCE completion rate was $67.9 \%$, sufficient to not terminate the trial. However, adjustments to the procedures will be pursued. The completion rate was lower than in previous studies [11-14], although in line with results from other screening populations $[4,15]$ and with a conclusive investigation rate of $80.3 \%$. The PDR was $73.5 \%$, whereas in the national CRC screening program, the adenoma detection rate (ADR) is $49 \%$. Six suspected cancers were found $(2.6 \%)$, whereas in the national CRC screening, the cancer rate is $4.2 \%$ [7]. The PDR ap- 
Original calculation

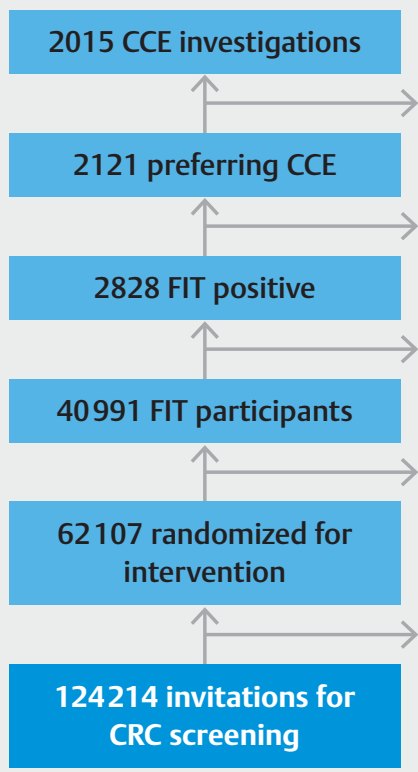

New sample size calculations

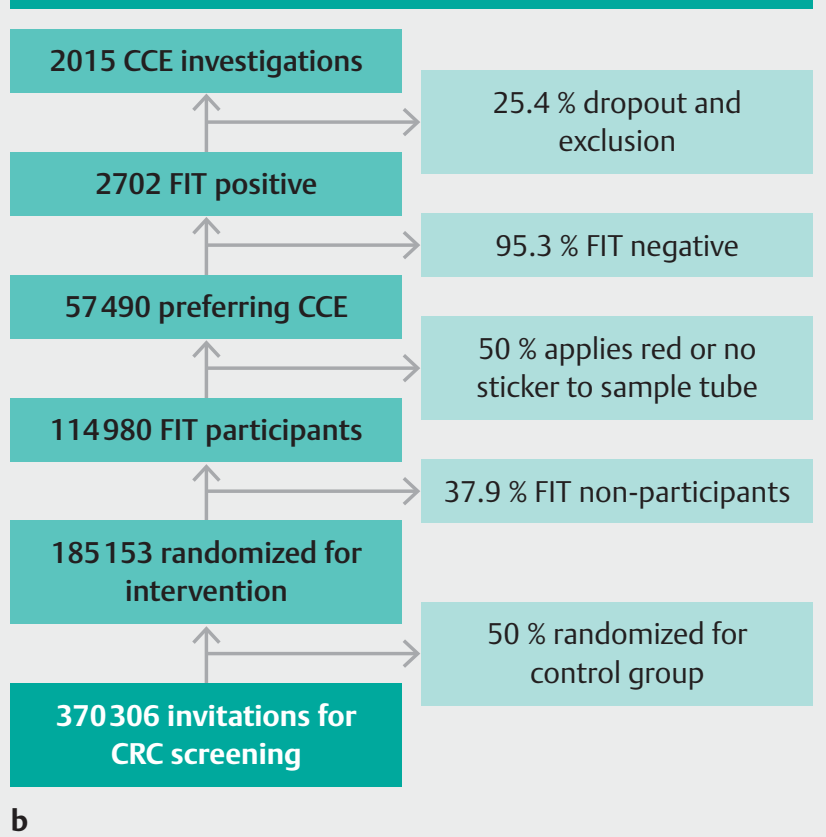

- Fig. 3 a Power flowchart of original calculations and $\mathbf{b}$ calculations applying adjustments for assumptions for dropout rate, preference proportion, FIT-positive rate, and FIT participation rate.

pears high from CCE, but the result should be interpreted with caution, as ADRs do not include hyperplastic polyps. Manual reading of CCE videos does not allow for confirmatory conclusions about polyp histology; therefore, ADRs from CCE cannot be calculated prior to pathological assessment. Further, pathological assessment cannot be completed in all CCE-detected polyps as not all of them are referred for polypectomy with ensuing pathological assessment. However, we have conducted a back-to-back study performing CCE and colonoscopy in 253 individuals from a screening population. The PDR was $74 \%$ for CCE and $64 \%$ for colonoscopy [4]. This aligns very well with the results of the current study. Inherent in the assessment of PDR from CCE will also be the risk of false positives, because the lesions are not resected, and therefore, not confirmed pathologically. False positives can occur either if a polyp finding is not a polyp or if the same polyp is identified more than once due to backwards progression of a capsule [16]. Whether the PDR is higher with CCE than with colonoscopy is therefore difficult to conclude. It seems, however, very unlikely that the ADR in the experimental group is significantly lower than in the general screening population.

Evaluating AEs showed that $12 \%$ of CCE patients experienced abdominal pain and $8.2 \%$ experienced rectal bleeding, while $19.6 \%$ described other minor AEs. These proportions are larger than those reported for colonoscopy.

A less than $1 \%$ rate of complications and a less than $0.5 \%$ rate of rectal bleeding is reported with colonoscopy $[17,18]$. However, these numbers are reports from registered complications in hospitals and minor AEs do not always lead to hospital- ization or hospital contacts. In a previous study, we asked 200 colonoscopy patients to fill in questionnaires about colonoscopy-related complications. When collecting information about these minor AEs systematically as PRO, 14\% experienced abdominal pain and 5\% experienced bleeding [19]. Further, in screening individuals undergoing both CCE and colonoscopy, $58.4 \%$ experienced medium-high levels of discomfort from colonoscopy, while 5.9\% did from CCE [3]. In Denmark, screening colonoscopies are performed in outpatient clinics, where patients leave the hospital within 2 hours after colonoscopy, and many minor complications will not be registered. Our complication rate might be higher than some comparisons, simply because we asked the patients.

No major AEs were recorded. This is in accordance with the results from a recent review reporting no CCE-related AEs in 13 included studies, of which eight introduced CCE as a filter test following positive FIT in screening populations [15]. The time spans from positive FIT until CCE and from CCE until CCE diagnostics were acceptable and within the time guarantee in the screening program. Technical issues were limited, as only one investigation was deemed unusable and one had transmission failure. In six instances in which the technical score was not reported, half were due to oral capsule excretion.

The assumptions made for sample size calculations had all been too optimistic, especially the assumed $6.9 \%$ FIT-positive rate and $5 \%$ dropout rate compared to $4.7 \%$ and $25.4 \%$ observed, which led to an underestimation of the needed sample size for randomization. The sample size, therefore, was adjusted to 370,306 individuals for randomization. This is probably 
an overestimation of the true number, because follow-up from the FIT invitation date was 8 weeks for individuals with the shortest follow-up and some might participate after followup. We know from previous analyses that for the first 2 years of screening in Denmark, $95 \%$ of individuals submitting a FIT sample did so within 42 days [20] and the overestimation, therefore, is probably limited.

Except for FIT participation and positive rates, it is not possible to compare results with the control group until the trial has been terminated, as written consent was not obtained from this group and the purpose of the interim analyses was to ensure efficient and safe procedures in the intervention group of the study. No such concerns are present for the control group, as they follow a standard screening trajectory. Compared to the national screening in general, the participation rate appears high in the intervention group, the PDR is high, and the detection rate for suspected cancer is low.

Forty percent of participants did not indicate their preference for CCE or colonoscopy. Because the information about the trial was added to an already extensive information package from the national screening program, it is possible that some potential participants did not read it. Furthermore, because this was the second round of screening in Denmark, some of the participants may previously have participated in screening and may therefore repeat the participation process from the earlier round, without reading the trial information. Therefore, it was decided to revise the patient information even though the participation rate was acceptable. In addition, we introduced a reminder to FIT-positive individuals in the intervention group who had not indicated their preference of diagnostic procedure. The Regional Health Research Ethics Committee has approved any revised or new patient information.

\section{Conclusions}

So far, CareForColon2015 has ensured efficient and safe participation. Participation rates and PDRs appear higher than in the general screening program, but the detection rate for suspected cancer is lower. The completion rate for CCE was acceptable, therefore, the study will not be terminated earlier than planned, but the CCE procedures were reviewed and $2 \mathrm{mg}$ of Resolor (prucalopride) was added to the regimen. Resolor will be administered 45 to 60 minutes prior to capsule ingestion and we will analyze the potential improvements after screening of 203 patients with CCE. The sample size has been adjusted to 370,306 individuals for randomization.

\section{Acknowledgements}

The CFC2015 trial received funding from Aage and Johanne Louis-Hansen's Fond (grant 17-2B-1409), OUH's innovation fund (grant: R75-A3392), Medtronic Research Foundation (grant ERP 2018-11151), the Danish Cancer Society (grant R100-A6747), and the Excellence Centre in the Region of Southern Denmark (grant 18/48426). Medtronic VR (Minneapolis, Minnesota, United States) provided the capsules, but had no influence on study protocol, analysis, or interpretation of the results.

\section{Competing interests}

The authors declare that they have no conflict of interest.

\section{References}

[1] Kaalby L, Deding U, Kobaek-Larsen M et al. Colon capsule endoscopy in colorectal cancer screening: A randomized controlled trial. BM] Open Gastroenterol 2020; 7: 1-7

[2] Groth S, Krause H, Behrendt R et al. Capsule colonoscopy increases uptake of colorectal cancer screening. BMC Gastroenterol 2012; 12 : $1-7$

[3] Thygesen MK, Baatrup G, Petersen C et al. Screening individuals' experiences of colonoscopy and colon capsule endoscopy; A mixed methods study. Acta Oncol (Stockholm, Sweden) 2019; 58: 1-6

[4] Kobaek-Larsen M, Kroijer R, Dyrvig AK et al. Back-to-back colon capsule endoscopy and optical colonoscopy in colorectal cancer screening individuals. Colorectal Dis 2018; 20: 479-485

[5] Den tværregionale implementeringsgruppe vedr. tarmkræftscreening. Screenings- og adenomkontrol program for tyk-og endetarmskræft, guidelines for koloskopi og patologi [Screening- and adenomacontrol program for colorectal cancer, guidelines for colonoscopy and pathology]. Danske Regioner; 2014

[6] Leighton JA, Rex DK. A grading scale to evaluate colon cleansing for the PillCam COLON capsule: A reliability study. Endoscopy 2011; 43: 123-127

[7] Dansk tarmkræftscreeningsdatabase årsrapport 2018 [Annual Report of the Danish Colorectal Cancer Screening Database 2020]. Database. DCCS; 2020

[8] Deding U, Henig AS, Salling A et al. Sociodemographic predictors of participation in colorectal cancer screening. Int J Colorectal Dis 2017; 32: $1117-1124$

[9] Deding U, Henig AS, Hindersson P et al. Determinants of non-participation in colon examination following positive stool sample in colorectal cancer screening. Eur J Public Health 2019; 29: 1118-1124

[10] Senore C, Basu P, Anttila A et al. Performance of colorectal cancer screening in the European Union Member States: Data from the second European screening report. Gut 2019; 68: 1232-1244

[11] Spada C, Pasha SF, Gross SA et al. Accuracy of first- and second-generation colon capsules in endoscopic detection of colorectal polyps: A systematic review and meta-analysis. Clin Gastroenterol Hepatol 2016; 14: 1533-1543

[12] Deding U, Kaalby L, Bøggild $\mathrm{H}$ et al. Colon capsule endoscopy vs. CT colonography following incomplete colonoscopy: A systematic review with meta-analysis. Cancers (Basel) 2020; 12: 1-17

[13] Kjølhede T, Ølholm AM, Kaalby L et al. Diagnostic accuracy of capsule endoscopy compared to colonoscopy for polyp detection: Systematic review and meta-analyses. Endoscopy 2021; 53: 713-721

[14] Vuik FER, Nieuwenburg SAV, Moen S et al. Colon capsule endoscopy in colorectal cancer screening: A systematic review. Endoscopy 2021: doi:10.1055/a-1308-1297

[15] Pioche M, Ganne C, Gincul R et al. Colon capsule versus computed tomography colonography for colorectal cancer screening in patients with positive fecal occult blood test who refuse colonoscopy: A randomized trial. Endoscopy 2018; 50: 761-769

[16] Deding U, Herp J, Havshoei AL et al. Colon capsule endoscopy versus CT colonography after incomplete colonoscopy. Application of artifi- 
cial intelligence algorithms to identify complete colonic investigations. United Gastroenterol J 2020; 8: 782-789

[17] Mikkelsen EM, Thomsen MK, Tybjerg J et al. Colonoscopy-related complications in a nationwide immunochemical fecal occult blood test-based colorectal cancer screening program. Clin Epidemiol 2018; 10: 1649-1655

[18] Reumkens A, Rondagh EJ, Bakker CM et al. Post-colonoscopy complications: A systematic review, time trends, and meta-analysis of population-based studies. Am J Gastroenterol 2016; 111: 1092-1101
[19] Steffenssen MW, Al-Najami I, Zimmermann-Nielsen E et al. Patientreported complications related to colonoscopy: A prospective feasibility study of an email-based survey. Acta Oncol 2019; 58: 65-70

[20] Deding U, Henig AS, Torp-Pedersen C et al. The effects of reminders for colorectal cancer screening: Participation and inequality. Int J Colorectal Dis 2019; 34: 141-150 